
\title{
28 Research Square \\ Efficacy and Safety of Apatinib in Patients with Recurrent or Refractory Melanoma
}

\author{
Shumin Yuan \\ Henan Cancer Hospital \\ Qiang Fu \\ Henan Cancer Hospital

\section{Yingkun Ren} \\ Henan Cancer Hospital

\section{Zhimeng Li} \\ Henan Cancer Hospital

\section{Huijuan Wang} \\ Henan Cancer Hospital

\section{Xiaojie Zhang} \\ Henan Cancer Hospital

\section{Hao Huang}

Henan Cancer Hospital

\section{Yonghao Yang}

Henan Cancer Hospital

\section{Yiman Shang}

Henan Cancer Hospital

\section{Quanli Gao}

Henan Cancer Hospital

\section{Zibing Wang ( $\nabla$ zlyywzb2118@zzu.edu.cn )}

Affiliated Cancer Hospital of Zhengzhou University \& Henan Cancer Hospital

\section{Research article}

Keywords: Melanoma, Targeted therapy, Apatinib, PFS, OS

Posted Date: October 5th, 2020

DOI: https://doi.org/10.21203/rs.3.rs-67706/v1

License: (c) (1) This work is licensed under a Creative Commons Attribution 4.0 International License. Read Full License 
Version of Record: A version of this preprint was published at The Oncologist on January 1st, 2022. See the published version at https://doi.org/10.1093/oncolo/oyab068. 


\section{Abstract}

Background: Prognosis of patients with metastatic malignant melanoma is very poor and partly due to high resistance to conventional chemotherapies. The study's objectives were to assess the activity and tolerability of apatinib, an oral small molecule anti-angiogenesis inhibitor, in patients with recurrent advanced melanoma.

Methods: This was a single-arm, single-center phase II trial. The primary endpoint was progression-free survival (PFS) and the secondary endpoints were objective response rate (ORR), disease control rate (DCR), and overall survival (OS). Eligible patients received at least one first-line therapy for advanced melanoma and experienced recurrence. Apatinib $(500 \mathrm{mg}$ ) was orally administered daily. Trail registration: Clinical Trials, ID: NCT03383237. Registered on 24 December 2017. URL of trail registry record: https://register.clinicaltrials.gov.

Results: Fifteen patients were included in the analysis. The median PFS was 4.0 months. There were two major objective responses, for a $13.33 \%$ response rate. Eleven patients had stable disease, with a DCR of $86.67 \%$. The median OS was 12.0 months. The most common clinically significant grade 3 or 4 toxicities included hypertension and canker sore. No treatment-related deaths occurred.

Conclusions: Apatinib showed antitumor activity as a second or first-line therapy in patients with malignant melanoma. The toxicity was manageable.

\section{Background}

Patients with advanced melanoma, dacarbazine alone, or in combination with other cytotoxic agents has been recommended as first-line treatment for the past 40 years; however, the objective response rate was only $15 \%$, without improvement insurvival (1). In recent years, the treatment has been revolutionized by advances in molecular-targeted therapy and immunotherapy. These treatments include BRAF and MEK kinase and immune checkpoint inhibitor anti-cytotoxic T-lymphocyte-associated cytotoxic T-lymphocyte antigen 4 antibody (anti-CTLA4) and anti-programmed cell death protein 1 antibody (anti-PD1). At the advanced-stage, anti-PD1 and anti-CTLA4 antibodies (such as nivolumab, pembrolizumab, and ipilimumab), and selective BRAF inhibitors (vemurafenib and dabrafenib) alone and/or in combination, with MEK inhibitors (cobimetinib and trametinib) showed promising results in clinical trials (2-10). In BRAF V600E melanoma, combining BRAF inhibitors, with MEK inhibitors, showed an obvious curative effect, which led to high response rates (70\%), a rapid response induction, and symptom control. The progression-free survival was approximately 12 months (11-12). Nivolumab and pembrolizumab are effective in BRAF inhibitor-resistant mutant melanoma (13-14). There is no recommended standard therapy for patients who donot respond to chemotherapy and molecular-targeted and immune checkpoint inhibitor therapies.

Apatinib is an oral, small-molecule tyrosine kinase inhibitor of vascular endothelial growth factor-2 (VEGFR-2) (15). It inhibits tyrosine kinases such as PDGFR- $\beta$, c-SRC, c-Kit, and MET, reducing tumor 
microvessel density and effectively blocking tumor cells $(16,17)$. It can also upregulate the expression of cell cycle inhibitor p21 and p27 and downregulate cyclin B1 and cdc2 (18). Apatinib has anti-tumor potential against many tumors types, including hepatocellular carcinoma, gastric, non-small cell lung, and breast cancers (19-21). To date, few studies on malignant melanoma treatment using apatinib exist. Therefore, our study investigated the anti-tumor effect of apatinib on malignant melanoma. Additionally, the treatment tolerability was evaluated.

\section{Methods}

\section{Study Design and Participants}

This study included patients from the Affiliated Cancer Hospital of Zhengzhou University. Inclusion Criteria: age, $\geq 18$ and $\leq 70$ years; ECOG PS, 0 or 1; Life expectancy, $\geq 3$ months; Adequate hepatic, renal, heart, and hematologic functions, ANC $\geq 1.5 \times 109 / \mathrm{L}, \mathrm{PLT} \geq 100 \times 109 / \mathrm{L}, \mathrm{HB} \geq 90 \mathrm{~g} / \mathrm{L}, \mathrm{TBIL} \leq 1.5 \times \mathrm{ULN}$, and ALT or AST $\leq 2.5 \times U L N$ (or $\leq 5 \times U L N$ in patients, with liver metastases), Serum $\mathrm{Cr} \leq 1.5 \times \mathrm{ULN}$ and $\mathrm{Cr}$ clearance $\geq 60 \mathrm{~mL} / \mathrm{min}$;Left ventricular ejection fraction (LVEF) $\geq$ lower limit of normal (50\%). Exclusion Criteria: uncontrollable hypertension, grade II above myocardial ischemia or infarction, poor arrhythmic control (including QTc interval: male $\geq 450 \mathrm{~ms}$ and female $\geq 470 \mathrm{~ms}$ ); a variety of factors affecting oral absorption (such as inability to swallow, nausea, vomiting, chronic diarrhea, intestinal obstruction, etc.); patients with gastrointestinal bleeding risk; coagulation dysfunction (INR> 1.5, PT>ULN + 4s, or APTT> 1.5 ULN), with bleeding tendency or ongoing thrombolysis or anti-blood coagulation treatment; long-term unhealed wounds or fractures; active bleeding, within 30 days after major surgery; intracranial metastasis; pregnant or lactating women; allergic to apatinib; severe liver and kidney dysfunction; the investigators believe there is any condition that may harm the subject or result in the inability to meet the research requirements or a concomitant disease that seriously endangers the patient's safety or affects the patient in completing the study.

All patients provided written informed consent before participation in the study.

\section{Procedures}

After verification of eligibility, patients received oral apatinib $500 \mathrm{mg}$, once daily, until any of the following occurred: disease progression, according to RECIST 1.1 definition, death, unacceptable toxicity, or withdrawal of consent for any reason. Clinical safety assessment, including medical history, physical examination, and laboratory testing, was performed every 6 weeks. Adverse events were graded, according to CTCAE. The primary endpoint was progression-free survival (PFS). Secondary endpoints included overall survival (OS), objective response rate (ORR), and disease control rate (DCR).

\section{Statistical analyses}


PFS was defined as the time from treatment initiation to either first disease progression or death from any cause. Patients alive at the time of analysis were censored at the date of last disease assessment. OS was measured from the treatment initiation to death (from any cause) dates. PFS and OS were estimated using the Kaplan-Meier method in each stratum. The univariate Cox proportional hazards regression model was used to identify independent prognostic factors. $P<0.05$ was considered statistically significant. The final data analysis was carried out on July 10, 2020.

\section{Results}

\section{Patient characteristics}

Between December 2017 and December 2019, 15 eligible patients were recruited into this study and analyzed. A CONSORT diagram is shown in supplementary Fig. 1. The summary and detailed demographics and baseline characteristics of the 15 patients are shown in Table 1 and supplementary Table 1, respectively. At the time of analysis, treatment was discontinued in 10 patients because of death.

Table 1

Demographics and patient baseline characteristics

\begin{tabular}{|ll|}
\hline Characteristic & $\begin{array}{l}\text { Patients } \\
\text { N(\%) }\end{array}$ \\
\hline Age & $7(46.67)$ \\
$>60$ & $8(53.33)$ \\
Sex & \\
Female & $5(33.33)$ \\
Male & $10(66.67)$ \\
ECOG PS & $15(100)$ \\
$0-1$ & \\
Treatment & $9(60.00)$ \\
After first-line & $3(20.00)$ \\
After second-line & $2(13.33)$ \\
After third-line & $1(6.67)$ \\
After forth-line & \\
\hline
\end{tabular}




\section{Efficacy}

Disease progression or death occurred in 14 of the 15 patients (93\%) at the time of data cutoff. The median PFS duration was 4.0 months (Fig. 1). The median OS was 12 months (Fig. 2). In the univariate analysis, several baseline factors (sex, age, subtypes, lactate dehydrogenase level, number of organ sites, with metastasis, and treatment lines) were not associated with either PFS or OS (supplementary Table 2). No complete response was obtained, but a partial response was observed in two patients (Fig. 3). The ORR was $13.33 \%$, and DCR was $86.67 \%$.

\section{Toxicity}

Table 2 summarizes all adverse events, which occurred in the participants. The most common grade $1 / 2$ adverse events were hypertension (80.00\%), hand or foot skin reaction $(26.67 \%)$, canker sore (33.33\%), liver function damage (20.00\%), hemorrhage (20.00\%), diarrhea (20.00\%), sick (13.33\%), anepithymia (13.33\%), rash (6.67\%), and fever (6.67\%). The most common grade $3 / 4$ adverse events were hypertension (6.67\%) and canker sores (6.67\%). No treatment-related deaths occurred.

Table 2

Adverse events in participants

\begin{tabular}{|llll|}
\hline \multicolumn{4}{|c|}{ Patients by Event Grade } \\
\hline Events & Total & G1 or G2 & G3 or G4 \\
& N (\%) & N (\%) & N (\%) \\
\hline Hypertension & $12(80.00 \%)$ & $11(73.33 \%)$ & $1(6.67 \%)$ \\
\hline Hand-foot skin reaction & $4(26.67 \%)$ & $4(26.67 \%)$ & 0 \\
\hline Canker sore & $5(33.33 \%)$ & $4(26.67 \%)$ & $1(6.67 \%)$ \\
\hline Liver function damage & $3(20.00 \%)$ & $3(20.00 \%)$ & 0 \\
\hline Hemorrhage & $3(20.00 \%)$ & $3(20.00 \%)$ & 0 \\
\hline Diarrhea & $3(20.00 \%)$ & $3(20.00 \%)$ & 0 \\
\hline Sick & $2(13.33 \%)$ & $2(13.33 \%)$ & 0 \\
\hline Anepithymia & $2(13.33 \%)$ & $2(13.33 \%)$ & 0 \\
\hline Rash & $1(6.67 \%)$ & $1(6.67 \%)$ & 0 \\
\hline Fever & $1(6.67 \%)$ & $1(6.67 \%)$ & 0 \\
\hline
\end{tabular}

\section{Discussion}


As a BRAF inhibitor, vemurafenibis is superior to chemotherapy concerning PFS, OS, and ORR, in BRAF mutant advanced melanoma patients (22). A second BRAF inhibitor, dabrafenib, exhibited a significantly way to improve PFS, compared to chemotherapy (23). Compared to BRAF inhibitors, the MEK inhibitors trametinib and binimetinib had lower ORR (20 vs $50 \%$ ); however, they were more superior, compared to chemotherapy in patients with BRAF-mutant advanced melanoma $(24,25)$. The majority of patients treated with BRAF or MEK inhibitors develop drug resistance. Combining BRAF and MEK inhibitors may overcome this limitation. The superiority of combining these two inhibitor categories, compared to singleagent inhibitor therapy, was confirmed in several randomized trials, with PFS rate of $19 \%$ and OS, $34 \%$ at 5 years of receiving dabrafenib plus trametinib (26); mPFS, 14.9 months and mOS, 33.6 months receiving encorafenib plus binimetinib (27); mPFS, 9.9 months and mOS, 22.5 months receiving vemurafenib plus cobimetinib (28); and mPFS, 10-14 months and mOS, about 24 months (29). BRAF and MEK inhibitor combination improves outcomes in melanoma patients, with high adverse event frequencies. Moreover, resistance occurs eventually. Because of this, other molecular-targeted strategies are also being studied, including the use of small-molecule tyrosine kinase inhibitors of VEGF.

In the present study, apatinib showed antitumor activity in patients with malignant melanoma, with median PFS and OS of 4.0 and 12.0 months, respectively. Univariate analysis showed that several baseline factors (sex, age, subtypes, lactate dehydrogenase level, number of organ sites, with metastasis, and treatment lines) were not associated with either PFS or OS. Although not as good as BRAF and MEK inhibitors, considering that the patients enrolled are on second-line treatment, the drug has certain prospects and is worthy of large-scale clinical studies in malignant melanoma.

The toxicity profile was generally consistent with prior results using apatinib in a phase I study, with the safety data of other multi-kinase inhibitors of the same class. The adverse events were hypertension, hand-foot skin reactions, canker sores, liver function damage, hemorrhage, diarrhea, sick, anepithymia, rash, and fever. Most of these adverse events were mildly graded. Only a small proportion of subjects reported grade $3 / 4$ events. Among these, one patient (6.67\%) had grade 3 hypertension and one (6.67\%), grade 4 canker sores.

Apatinib efficacy and safety in this study were consistent with a prospective phase I study launched by Guo's team (30). In that study, 12 patients were treated with various apatinib doses (250 or $500 \mathrm{mg}$ daily) plus temozolomide (100 or $200 \mathrm{mg}$ ). Among them, 1 patient achieved PR and 9 achieved SD. The ORR was $8.3 \%$ and DCR was $83 \%$. mPFS was 3.3 months and mOS 6.3 months. Regarding safety, doselimiting toxicities were not observed even in the temozolomide $300 \mathrm{mg}$ plus apatinib $500 \mathrm{mg}$ daily group. In a retrospective analysis of 22 patients treated, with $500 \mathrm{mg}$ apatinib per day, ORR was $9.1 \%$ and DCR $59.1 \%$. The mPFS was 7.5 months (31). The common feature of these two studies and ours is that the patients enrolled are Chinese, with mainly malignant melanomas of the mucosa and extremities. These two types of malignant melanoma have a low BRAF gene mutation rate; therefore, they may not be sensitive to BRAF inhibitors and PD-1 antibodies (32). Therefore, anlotinib may have better application prospects in this population. 
The present study had some limitations. When the IIT study was designed, vemurafenib or PD-1 mAb had not been approved for use in Chinese melanoma patients, and dacarbazine-based chemotherapy was the first-line treatment. Therefore, the initial inclusion criteria consisted of treating chemotherapy-refractory melanoma patients with first-line therapy. However, with vemurafenib and PD-1 approval in melanoma in China subsequently, patients had more standard choices. It became difficult to enroll new patients, who receive only the first-line treatment. After careful discussion, the study investigators and sponsor revised our regimen, according to the applicable regulations, protecting the rights, safety, and welfare of subjects. Consent was obtained from patients with second-line or above treatment. Eventually, 15 patients were analyzed in the study, among which 9 had first-line treatment and 6 had second-line or above treatment. Additionally, the patients enrolled in this study were from China. The generalizability to other populations remains unclear. Finally, due to the small sample size of this study, the results we have obtained on patient prognosis are not significantly correlated, with baseline data and need to be interpreted with caution.

\section{Conclusions}

In conclusion, apatinib had antitumor activity in patients with metastatic melanoma second-line or greater treatment. The toxicity was manageable and acceptable.

\section{Abbreviations}

PFS: progression-free survival; mPFS: median progression-free survival; ORR: objective response rate; DCR: disease control rate; OS: overall survival; mOS: median OS; anti-CTLA4: cytotoxic T-lymphocyte antigen 4 antibody; anti-PD1: anti-programmed cell death protein 1 antibody; VEGFR-2: vascular endothelial growth factor-2.

\section{Declarations}

\section{Ethics approval and consent to participate}

The study protocol for human studies was approved by the IRB of the Affiliated Cancer Hospital of Zhengzhou University. All participants gave informed written consent.

\section{Consent for publication}

All authors have agreed to publish this manuscript.

\section{Availability of data and material}


The datasets used and/or analyzed during the current study are available from the corresponding author on reasonable request.

\section{Competing interests}

The authors declare that they have no competing interests.

\section{Funding}

This work was supported by the National Natural Science Foundation of China (Grant No. 81972690 , 81000914 , and 81272526 ). The funding bodies played no role in the design of the study and collection, analysis, and interpretation of data and in writing the manuscript.

\section{Authors' contributions}

SY analyzed the data and wrote the manuscript. QF, YR, ZL, HW, XZ, HH, YY, YS and QG assisted in sample collection and response evaluation in some patients. ZW designed and supervised the study. All authors have read and approved the manuscript.

\section{Acknowledgements}

We are thankful to the investigators and patients, who enrolled in this clinical trial.

\section{Affiliations}

${ }^{1}$ Department of Immunotherapy, ${ }^{2}$ Department of General Surgery, ${ }^{3}$ Department of Internal Medicine, Affiliated Cancer Hospital of Zhengzhou University \& Henan Cancer Hospital, Zhengzhou, China.

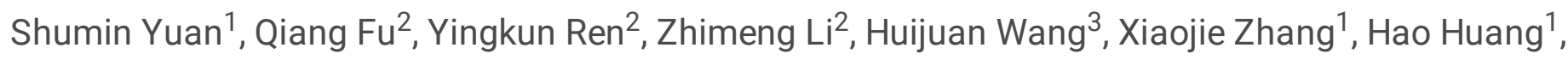
Yonghao Yang ${ }^{1}$, Yiman Shang ${ }^{1}$, Quanli Gao ${ }^{1}$, Zibing Wang ${ }^{1}$

\section{References}

1. Rastrelli M, Tropea S, Rossi CR, Alaibac M. Melanoma:epidemiology, risk factors, pathogenesis, diagnosis and classification. In Vivo. 2014;28:1005-1011.

2. Hodi FS, O'Day SJ, McDermott DF, Weber RW, Sosman JA, Haanen JB, et al. Improved survival with ipilimumab in patients with metastatic melanoma. N Engl J Med. 2010;363:711-723. 
3. Hauschild A, Grob JJ, Demidov LV, Jouary T, Gutzmer R, Millward M, et al. Dabrafenib in BRAFmutated metastatic melanoma: A multicentre, open-label, phase 3 randomised controlled trial. Lancet. 2012;380:358-65.

4. McArthur GA, Chapman PB, Robert C, Larkin J, Haanen JB, Dummer R, et al. Safety and efficacy of vemurafenib in BRAF(V600E) and BRAF(V600K) mutation-positive melanoma (BRIM-3): Extended follow-up of a phase 3, randomised, open-label study. Lancet Oncol. 2014;15: 323-32.

5. Robert C, Long GV, Brady B, Dutriaux C, Maio M, Mortier L, et al. Nivolumab in previously untreated melanoma without BRAF mutation. N Engl J Med. 2015;372: 320-30.

6. Robert C, Karaszewska B, Schachter J, Rutkowski P, Mackiewicz A, Stroiakovski D, et al. Improved overall survival in melanoma with combined dabrafenib and trametinib. N Engl J Med. 2015;372: 309.

7. Smalley KS, Sondak VK. Inhibition of BRAF and MEK in BRAF-mutant melanoma. Lancet.2015 Aug 1;386(9992):410-2.

8. Ribas A, Puzanov I, Dummer R, Schadendorf D, Hamid O, Robert C, et al. Pembrolizumab versus investigator-choice chemotherapy for ipil- imumab-refractory melanoma (KEYNOTE-002): A randomised, controlled, phase 2 trial. Lancet Oncol. 2015;16: 908-918.

9. Weber JS, D'Angelo SP, Minor D, Hodi FS, Gutzmer R, Neyns B, et al. Nivolumab versus chemotherapy in patients with advanced melanoma who progressed after anti-CTLA-4 treatment (CheckMate 037): A randomised, controlled, open-label, phase 3 trial. Lancet Oncol. 2015;16: 375-384.

10. Ascierto PA, McArthur GA, Dréno B, Atkinson V, Liszkay G, Di Giacomo AM, et al. Cobimetinib combined with vemurafenib in advanced BRAF(V600)-mutant melanoma (coBRIM): Updated efficacy results from a randomised, double-blind, phase 3 trial. Lancet Oncol. 2016;17: 1248-1260.

11. Robert C, Karaszewska B, Schachter J, Rutkowski P, MackiewiczA, Stroiakovski D, et al. Improved overall survival in melanoma with combined dabrafenib and trametinib. N Engl J Med. 2015;372: 3039.

12. Long GV, Stroyakovskiy D, Gogas H, Levchenko E, de Braud F, Larkin J, et al. Dabrafenib and trametinib versus dabrafenib and placebo for Val600 BRAF-mutant melanoma: A multicentre, doubleblind, phase 3 randomised controlled trial. Lancet. 2015; 386: 444-451.

13. Mangana J, Cheng PF, Schindler K, Weide B, Held U, Frauchiger AL, et al. Analysis of BRAF and NRAS mutation status in advanced melanoma patients treated with anti-CTLA 4 antibodies: Association with overall survival? PLoS One, 2015;10:e013943810.

14. Robert C, Schachter J, Long GV, Arance A, Grob JJ, Mortier L, et al. KEYNOTE-006 investigators: Pembrolizumab versus ipilimumab in advanced melanoma. N Engl J Med. 2015; 372: 2521-32.

15. Tian S, Quan H, Xie C, Guo H, Lü F, Xu Y, et al.YN968D1 is a novel and selective inhibitor of vascular endothelial growth factor receptor-2 tyrosine kinase with potent activity in vitro and in vivo. Cancer Sci. 2011;102:1374-80.

16. Tong XZ, Wang F, Liang S, Zhang X, He JH, Chen XG, et al. Apatinib (YN968D1) enhances the efficacy of conventional chemotherapeutical drugs in side population cells and ABCB1-overexpressing 
leukemia cells. Biochem Pharmacol. 2012;83:586-97.

17. Geng R, Li J. Apatinib for the treatment of gastric cancer. Expert Opin Pharmacother. 2015;16:117-22

18. Yang C, Qin S. Apatinib targets both tumor and endothelial cells in hepatocellular carcinoma. Cancer Med. 2018;7:4570-4583.

19. Gomila A, Carratalà J, Badia J, Camprubí D, Piriz M, Shaw E, et al. Preoperative oral antibiotic prophylaxis reduces pseudomonas aeruginosasurgical site infections after elective colorectal surgery: a multicenter prospective cohort study. BMC Infect Dis. 2018;18:507.

20. Li J, Qin S, Xu J, Xiong J, Wu C, Bai Y, et al. Randomized, double-blind, placebo-controlled phase III trial of apatinib in patients with chemotherapy-refractory advanced or metastatic adenocarcinoma of the stomach or gastroesophageal junction. J Clin Oncol. 2016;34:1448-54.

21. Wang L, Liang L, Yang T, Qiao Y, Xia Y, Liu L, et al. A pilot clinical study of apatinib plus irinotecan in patients with recurrent high-grade glioma: clinical trial/experimental study. Medicine (Baltimore). 2017;96:e9053.

22. Chapman PB, Hauschild A, Robert C, Haanen JB, Ascierto P, Larkin J, et al. Improved Survival With Vemurafenib in Melanoma With BRAF V600E Mutation. N EnglJMed. 2011;364:2507-16.

23. Hauschild A, Grob JJ, Demidov LV, Jouary T, Gutzmer R, Millward M, et al. Dabrafenib in BRAFmutated Metastatic Melanoma: A Multicentre, Open-Label, Phase 3 Randomised Controlled Trial. Lancet. 2012;380:358-65.

24. Flaherty KT, Robert C, Hersey P, Nathan P, Garbe C, Milhem M, et al. Improved Survival With MEK Inhibition in BRAF-mutated Melanoma. N Engl J Med. 2012;367:107-14.

25. Ascierto PA, Schadendorf D, Berking C, Agarwala SS, van Herpen CM, Queirolo P, et al. MEK162 for patients with advanced melanoma harbouring NRAS or Val600 BRAF mutations: a non-randomised, open-label phase 2 study. Lancet Oncol. 2013;14:249-56.

26. Robert C, Grob JJ, Stroyakovskiy D, Karaszewska B, Hauschild A, Levchenko E, et al. Five-Year Outcomes With Dabrafenib Plus Trametinib in Metastatic Melanoma. N Engl J Med. 2019;381:626636.

27. Dummer R, Ascierto PA, Gogas HJ, Arance A, Mandala M5, Liszkay G, et al. Overall Survival in Patients With BRAF-mutant Melanoma Receiving Encorafenib Plus Binimetinib Versus Vemurafenib or Encorafenib (COLUMBUS): A Multicentre, Open-Label, Randomised, Phase 3 Trial. Lancet Oncol. 2018;19:1315-1327.

28. Ascierto PA, McArthur GA, Dréno B,Atkinson V, Liszkay G, Di Giacomo AM, et al. Cobimetinib Combined With Vemurafenib in Advanced BRAF(V600)-mutant Melanoma (coBRIM): Updated Efficacy Results From a Randomised, Double-Blind, Phase 3 Trial. Lancet Oncol. 2016;17:1248-60.

29. Ugurel S, Röhmel J, Ascierto PA, Flaherty KT, Grob JJ, Hauschild A, et al. Survival of Patients With Advanced Metastatic Melanoma: The Impact of Novel Therapies-Update 2017. Eur J Cancer. 2017;83:247-257.

30. Yang L, Zhu H, Luo P, Chen S, Xu Y, Wang C. Safety and Efficacy of Apatinib Combined With Temozolomide in Advanced Melanoma Patients After Conventional Treatment Failure. Transl Oncol. 


\section{8;11:1155-1159.}

31. Yang L, Zhu H, Luo P, Chen S, Xu Y, Wang C. Apatinib Mesylate Tablet in the Treatment of Advanced Malignant Melanoma. Onco Targets Ther. 2018;11:5333-5338.

32. Shoushtari AN, Munhoz RR, Kuk D, Ott PA, Johnson DB,Tsai KK, et al. The Efficacy of anti-PD-1 Agents in Acral and Mucosal Melanoma. Cancer. 2016;122:3354-3362.

Figures

\section{Survival proportions: Survival of pfs}

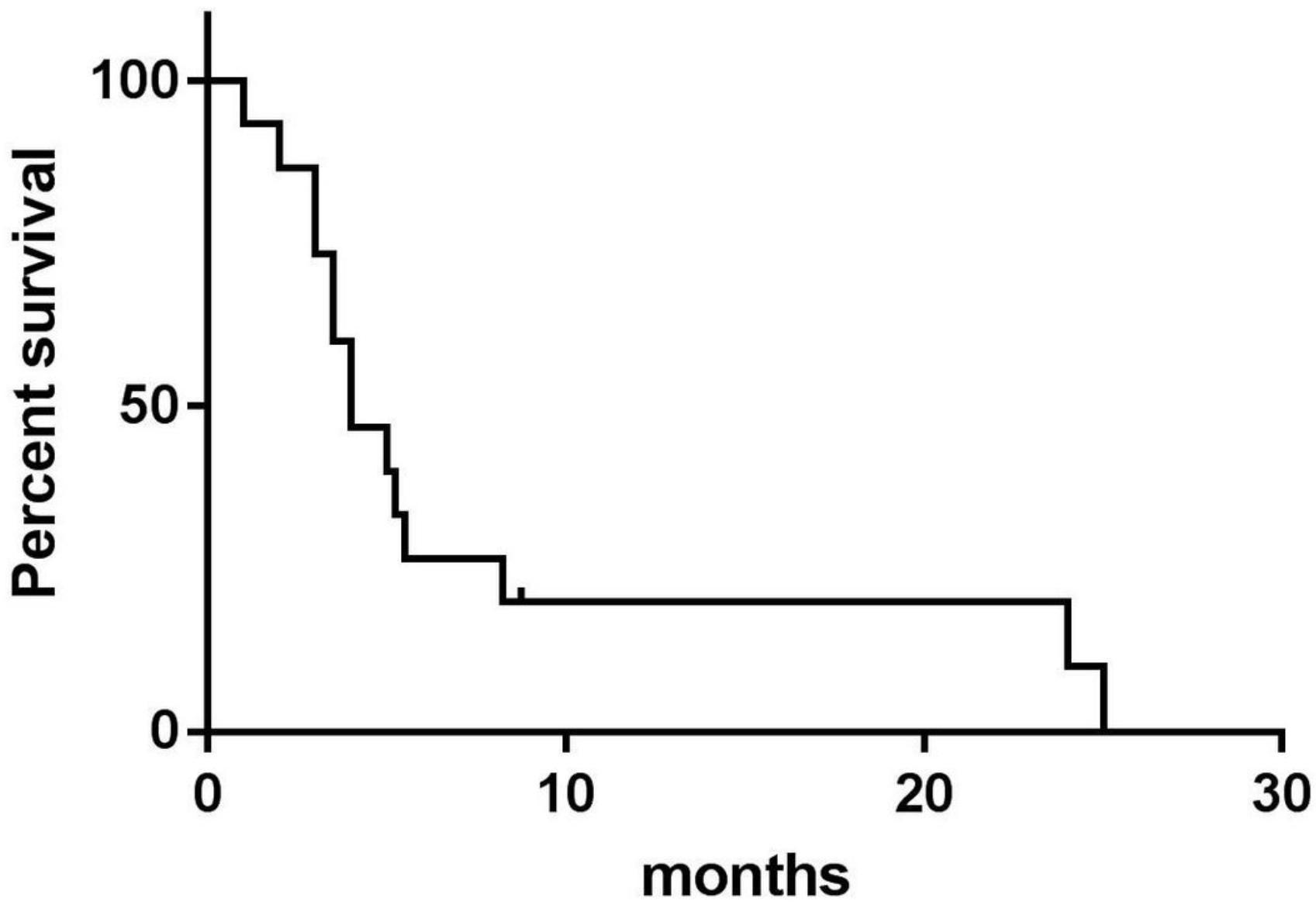

Figure 1

Kaplan-Meier curve for PFS. 


\section{Survival proportions: Survival of os}

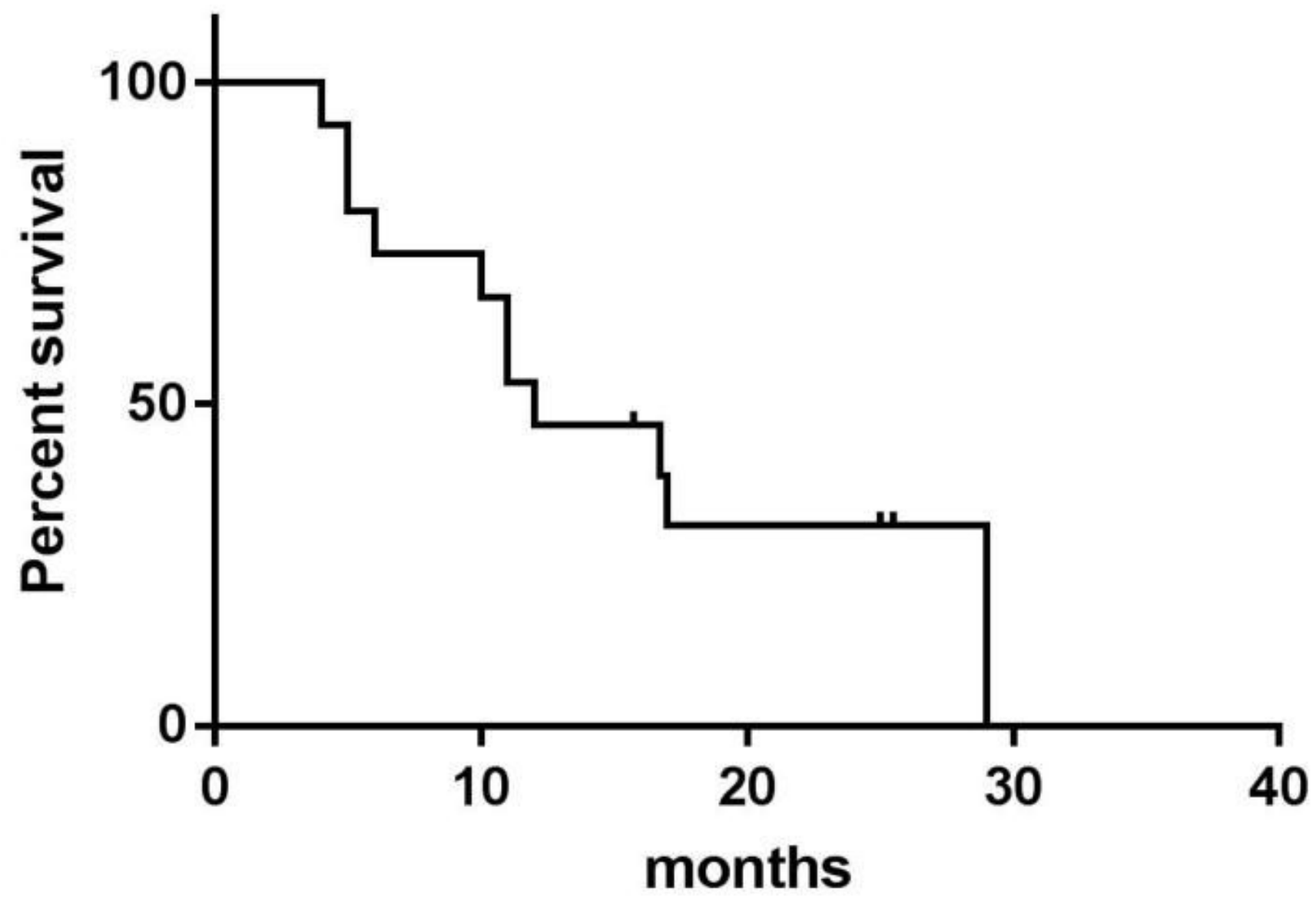

Figure 2

Kaplan-Meier curve for OS. 


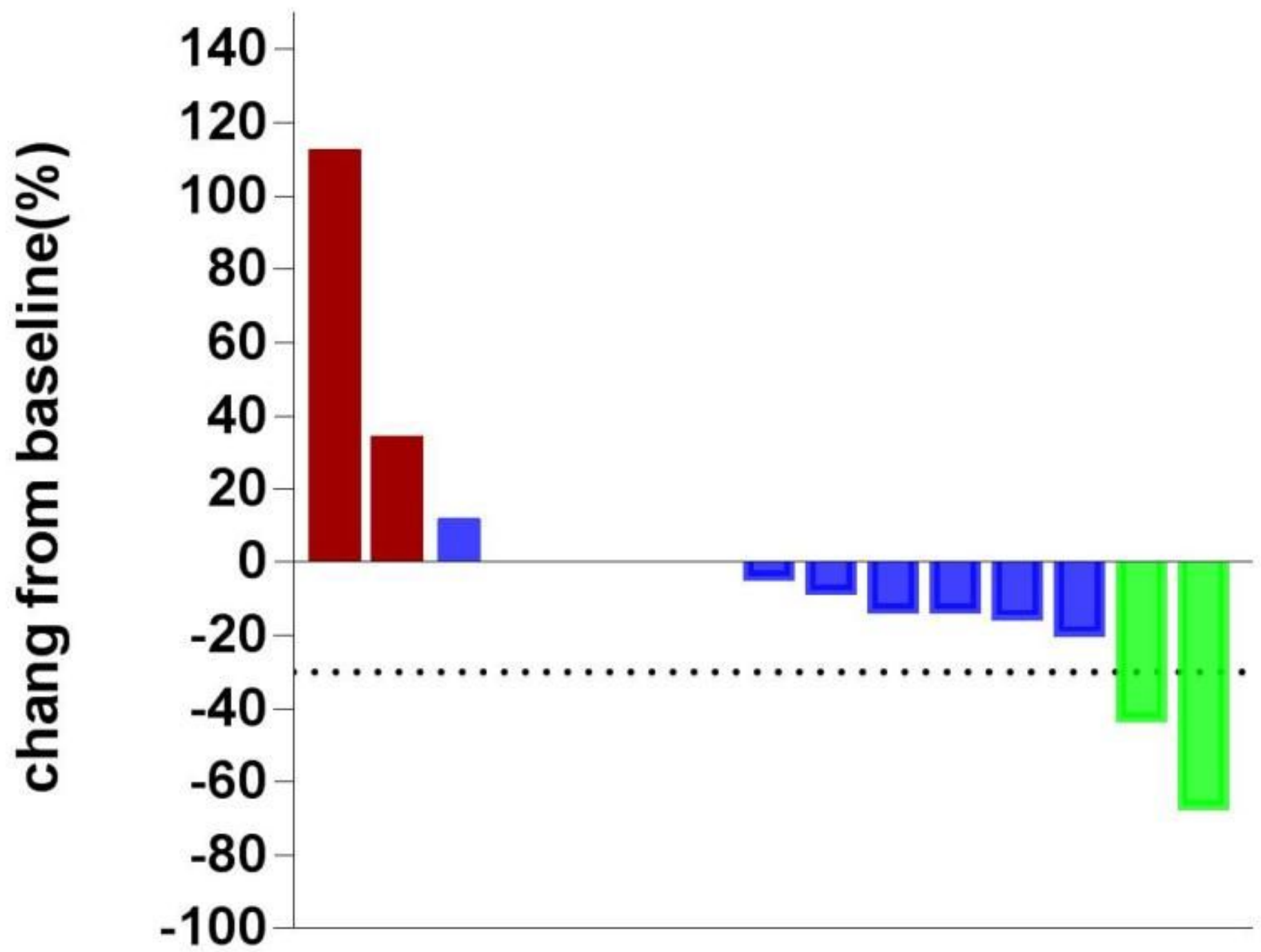

Figure 3

The waterfall plot for the best percentage change in target lesion size.

\section{Supplementary Files}

This is a list of supplementary files associated with this preprint. Click to download.

- SupplementaryTable1.docx

- supplementaryfigure1.jpg

- SupplementaryTable2.docx 